\title{
ATENÇÃO FARMACÊUTICA NAS INTOXICAÇÕES MEDICAMENTOSAS
}

\author{
Danniele do Espírito Santo Dantas ${ }^{1}$ \\ Leonardo Guimarães de Andrade ${ }^{2}$ \\ Sebastian Rinaldi Neto ${ }^{3}$
}

RESUMO: O uso de medicamentos pode ser considerado a principal causa das intoxicações relatadas no Brasil, sendo também um importante causa de morbimortalidade em todo o mundo e o principal motivo de internação. A gravidade e o impacto são decorrentes de fatores como a dose e o tipo de medicamento a que o indivíduo está exposto. $\mathrm{O}$ abuso e o uso irracional de drogas representam um risco significativo para a saúde pública, pois representam cerca de $30 \%$ dos casos de intoxicação. Nesse sentido, a prática da automedicação é um agravante do quadro geral de intoxicações no Brasil. Portanto, a promoção do uso racional de medicamentos e o monitoramento do tratamento medicamentoso por meio da assistência farmacêutica constitui uma importante ferramenta para redução de lesões e riscos à saúde do paciente, consequentemente, diminuir os casos de intoxicações. Nesse caso, vale ressaltar que a intoxicação por drogas é um grave problema de saúde pública, a disponibilidade de medicamentos, as práticas de automedicação e a qualidade dos serviços de saúde estão diretamente relacionados a esse problema. Portanto, é necessário adotar medidas preventivas e educacionais para reduzir as ocorrências de intoxicações e minimizar essas consequências. Nesse sentido, o farmacêutico desempenha um papel importante, reproduzindo funções efetivas em todas as áreas de atividade, colaborando com as práticas educativas e promovendo o uso racional dos medicamentos, garantindo assim o bem-estar das pessoas.

Palavras-chave: Atenção farmacêutica. Uso racional de medicamentos. Intoxicação medicamentosa. Automedicação.

ABSTRACT: The use of medications can be considered the main cause of poisoning reported in Brazil, it is also an important cause of morbidity and mortality worldwide and the main reason for hospitalization. The severity and impact are due to factors such as the dose and type of medication to which the individual is exposed. Abuse and irrational use of drugs represent a significant risk to public health, as they represent around $30 \%$ of cases of intoxication. In this sense, the practice of self-medication is an aggravating factor in the general situation of poisoning in Brazil. Therefore, promoting the rational use of medication and monitoring drug treatment through

\footnotetext{
I (Unig - Universidade Iguaçu, Curso de Graduação em Farmácia, Nova Iguaçu-Rj, Brasil).

${ }^{2}$ Mestre em Ciências do Meio Ambiente ( Universidade Veiga de Almeida).

Mestre em Doenças Paraíba ( Universidad Autônoma de Asuncíon).

${ }^{3}$ Mestre em Ciências Biológicas (Farmacologia e Química Medicinal) - Universidade Federal do Rio de Janeiro (UFRJ).
} 
pharmaceutical assistance constitutes an important tool for reducing injuries and risks to the patient's health, consequently reducing cases of intoxication. In this case, it is noteworthy that drug intoxication is a serious public health problem, the availability of medicines, self-medication practices and the quality of health services are directly related to this problem. Therefore, it is necessary to adopt preventive and educational measures to reduce the occurrence of poisoning and minimize these consequences. In this sense, the pharmacist plays an important role, reproducing effective functions in all areas of activity, collaborating with educational practices and promoting the rational use of medicines, thus ensuring people's well-being.

Keywords: Pharmaceutical care. Rational use of medications. Drug intoxication. Selfmedication.

\section{INTRODUÇÃO}

Drogas são medicamentos elaborados com a finalidade de prevenção, tratamento, cuidados paliativos e até diagnóstico, porém, o uso excessivo e irracional pode prejudicar a saúde dos pacientes e, em muitos casos, levar à morte.

O sucesso da terapia medicamentosa é que alcançamos os resultados esperados, como prevenção, controle e cura de doenças, alívio dos sintomas e normalização dos parâmetros fisiológicos ou laboratoriais (CARVALHO, 2017; CORRER et al., 20II).

As Interações Medicamentosas são consideradas um importante problema de saúde pública, que pode ser acidental e intencional, causado por substâncias lícitas ou ilícitas, sendo é um importante causa de morbimortalidade no contexto global. Seu surgimento está relacionado a uma variedade de processos fisiológicos e patológicos, relacionados ao desempenho dos efeitos tóxicos da interação de substâncias endógenas ou exógenas, caracterizando-se por desequilíbrios fisiológicos e alterações bioquímicas no organismo (RANGEL \& FRANCELINO, 2018).

Uma atividade importante para diminuir a ocorrência de intoxicações por drogas exógenas é a assistência farmacêutica. $O$ objetivo é conscientizar as pessoas sobre o uso racional de medicamentos, ou seja, monitorar mais a sério o público sobre o tratamento medicamentoso, fornecer informações para evitar a automedicação, o uso também doses e doses baixas Muito grandes, muita frequência e concentração, e para determinar prescrições médicas inadequadas, portanto, é necessário encontrar profissionais qualificados para garantir a patologia correta, e o mais importante é evitar o uso de drogas, inclusive tentativas de suicídio (LEITE, et al, 2008). 
Como profissionais de saúde, o farmacêutico desempenha um papel importante na orientação e orientação da clientela e no uso uniforme dos medicamentos. Como a sociedade considera a farmácia a primeira opção para as situações médicas, é necessário esclarecer que o uso de medicamentos incertos pode causar sérios danos à saúde (ROCHA, 20II).

$\mathrm{Na}$ assistência farmacêutica, a consulta de saúde ainda é uma ferramenta importante que leva ao uso coeso de medicamentos. Orientando toda a sociedade para o uso uniforme de drogas e seus motivos incidentais, seguindo as orientações e informações transmitido pelos profissionais de saúde (ROCHA, 20II).

Segundo a Organização Mundial da Saúde (OMS), a intoxicação é um grave problema de saúde pública global, com cerca de 500 mil pessoas afetadas a cada ano e estima-se que $1,5 \%$ a $3 \%$ da população sejam envenenadas a cada ano. A maior causa das intoxicações, principalmente infantis, são os produtos de desinfecção, limpeza doméstica, cosméticos, farmacêuticos e químicos industriais (CARVALHO, 2017).

A droga corresponde à principal causa de intoxicação relatada e é a segunda causa de morbimortalidade associada à intoxicação humana (MATHIAS et al, 2019).

Nesse contexto, o estudo tem como objetivo descrever o perfil do Intoxicação Medicamentosa no Brasil, enfatizando a atuação do farmacêutico na prevenção desse agravo.

\section{OBJETIVO GERAL}

Descrever o perfil das intoxicações medicamentosas, uso indiscriminado de medicamentos e automedicação e avaliar o papel do farmacêutico na prevenção desse problema.

\section{OBJETIVOS ESPECÍFICOS}

- Apresentar a atenção farmacêutica nas intoxicações por automedicação;

- Descrever a importância do Sistema de Notificação de Intoxicações do Brasil;

- Determinar os fatores de risco que levam ao envenenamento por drogas;

- Relatar os cuidados farmacológicos da intoxicação por automedicação; 
- Descrever a importância do farmacêutico na orientação do uso racional de medicamentos e prevenção de intoxicações por medicamentos.

\section{JUSTIFICATIVA}

O fato da automedicação estender-se no mundo, resultando-se em um transtorno de saúde pública. Essa tal prática pode por um lado, de certa maneira, acarretar vantagens ao desafogar a grande procura do tão entediado Sistema Único de Saúde (SUS), já por outro, pode ocasionar graves risco à saúde, que vão a começar das mais básicas reações adversas até as mais complexas intoxicações.

\section{METODOLOGIA}

O presente artigo constitui-se, inicialmente, de um amplo levantamento bibliográfico, elaborado a partir de material já publicado, que foi selecionado de artigos científicos. Para a seleção dos artigos que se adotou a técnica da revisão de literatura, através de dados eletrônicos de artigos científicos, revista científica que relatassem sobre intoxicações por medicamentos. Essas informações foram encontradas em plataformas como Scielo, Google acadêmico, Conselho Federal de Farmácia (CFF), Ministério da saúde, Agência Nacional de Vigilância Sanitária (ANVISA) entre outros.

\section{REVISÃO DE LITERATURA}

\section{INTOXICAÇÃO MEDICAMENTOSA}

A Intoxicação Medicamentosa (IM) constitui-se por uma série de sinais e sintomas clínicos, tóxicos e bioquímicos causados pelo uso de medicamentos em doses acima das recomendadas para o tratamento clínico. Pode, ainda, ser definida como um conjunto de alterações fisiopatológicas toxicas com alterações bioquímicas, incentivado pela interação de um xenobiótico com o sistema biológico, resultando em um estado patológico (CAIRES et al., 2018).

Os medicamentos quando administrados de forma racional e respeitando o esquema terapêutico, pode ser considerado de grande importância para a manutenção 
da saúde do ser humano. Isto se torna de grande importância, visto que todas as pessoas, em alguma fase da vida, irão fazer o uso de algum tipo de medicamento (SILVA \& OLIVEIRA, 20I8; VIEIRA \& CAVEIÃO, 2016).

A solução da terapia medicamentosa depende de variáveis:

(I) o paciente tem acesso a terapia selecionada de acordo com as suas urgências fisiopatológicas;

(II) o paciente percebe e cumpri o regime terapêutico;

(III) a terapia selecionada é efetiva e possui propósitos terapêuticos préestabelecidos;

(IV) os medicamentos não geram danos à saúde do paciente.

Se alguma falha acontecer em algumas dessas condições podem comprometer a saúde e a qualidade de vida do paciente, por isso o torna-se uma necessidade para potencializar os benefícios e reduzir os danos causados pelo uso abusivo e irracional (SILVA \& OLIVEIRA, 20I8; CORRER et al., 20II).

As Interações Medicamentosas avançam principalmente em crianças menores de 5 anos, e 76\% dos casos são por ingestão acidental. As embalagens chamativas, o sabor adocicado e a coloração são uns dos principais elementos, que contribuem para ocorrência de casos de intoxicações acidentais em crianças (SILVA, 2016).

Os medicamentos representam uma ferramenta essencial nos sistemas de saúde, porém seu uso irracional e abusivo, sem a orientação de um profissional da saúde qualificado pode representar um grave risco à saúde. Dados da OMS apontam que a metade dos medicamentos comercializados são prescritos e dispensados de forma incorreta, além disso, a grande maioria dos pacientes utilizam de forma inadequada.

Quando esse consumo supera os níveis necessários ou devidamente prescritos, as consequências podem ser graves, de modo a causar agravos irreversíveis ou até mesmo levar a morte. Na maioria dos casos, esses agravos são: reações adversas, interações medicamentosas e, principalmente, toxicidade (SILVA, 2016).

Com base nessa realidade foram criados centros habilitados para notificar, orientar e acompanhar esses pacientes. Dentre eles destacamos os Centros de Informações e Assistência Toxicológicas (Centros de Informação Toxicológica CIATOX/CEATOX) (Sistema Nacional de Informações Tóxico-Farmacológicas- 
SINITOX), onde o CIATOX coleta as informações e repassa para o SINITOX, que organiza em um banco de dados nacional, analisa e dispersar as informações sobre os casos de intoxicações (RANGEL \& FRANCELINO, 2018).

Dados do Sistema Nacional de Informações Tóxico-Farmacológicas SINITOX (2010) registraram que das intoxicações gerais, 28\% corresponde a Interações Medicamentosas, e desses, 16,59\% produzido o óbito da vítima. Esse diagnóstico, demonstra a necessidade de uma melhor gestão das autoridades públicas e sanitárias acerca dos medicamentos, a fim de estimular e implantar uma política de educação em saúde e estratégias institucionais que venham a alterar essa realidade evidenciada (RANGEL \& FRANCELINO, 2018; CARVALHO, 2017).

\section{SISTEMA DE NOTIFICAÇÃO DE INTOXICAÇÕES NO BRASIL}

O contínuo desenvolvimento da indústria farmacêutica brasileira está relacionado ao baixo nível de escolaridade da população e ao fácil contato facial com essas drogas, levando a um aumento significativo nas taxas de Intoxicação Medicamentosa devido ao abuso e uso indevido de drogas. Portanto, é necessário notificar todos os incidentes relacionados ao uso e abuso de drogas (MONTE et al., 2016).

No Brasil, as informações sobre Intoxicação Medicamentosa são fornecidas pelo (SINITOX), sistema criado pelo Ministério da Saúde (MS) em 1980, seguindo o modelo norte-americano da American Association of Poison Control Centers (AAPCC - Associação Americana de Centros de Controles de Intoxicações). A principal responsabilidade do SINITOX é coordenar todo o processo de coleta, registro, análise e liberação dos casos de intoxicação registrados no Brasil. Esses dados de intoxicações são registrados pelo CIATOX em cada estado e anexados ao banco de dados de notificações, e posteriormente essas informações registradas são repassadas à ANVISA, e a mesma posteriormente repassa ao SINITOX (CARVALHO, 2017; FIOCRUZ, 20II).

Segundo dados do SINITOX, os medicamentos são as principais causas de intoxicação humana, sendo os benzodiazepínicos, antigripais, antidepressivos e anti- 
inflamatórios os mais comumente encontrados nos casos de Intoxicação Medicamentosa no Brasil (CAIRES et al., 2018).

Segundo pesquisa do SINITOX, nos casos de intoxicações notificados em 2010, o infarto do miocárdio foi responsável por cerca de $28 \%$. Os efeitos colaterais causados pelo uso excessivo podem aparecer em curto a longo prazo, levando à dependência e danos a órgãos vitais (CHAVES et al., 2017).

Os medicamentos é uma importante ferramenta para reduzir à incidência das doenças da população, razão pela qual seu desenvolvimento nas clínicas médicas atuais é um fato inegável. Embora esses recursos terapêuticos sejam inevitáveis no combate a diversas doenças, eles também estão em constante evolução, portanto, estão diretamente relacionados ao aumento da incidência de reações adversas a medicamentos, como por exemplo, as Reações Adversas a Medicamentos (RAM) e as Intoxicações Medicamentosas (IM) (SANTOS \& BOING, 2018).

De acordo com a Organização Mundial de Saúde, quaisquer eventos clínicos adversos que possam ocorrer durante o tratamento medicamentoso são considerados eventos adversos a medicamentos. Dentre esses eventos adversos, podemos destacar as Reações Adversas a Medicamentos (RAM) e as Intoxicações Medicamentosas (IM), ambas causadas pelo uso de medicamentos, mas a diferença entre elas está na dosagem utilizada. A dose usada é terapêutica ou comumente, geralmente reage de forma não intencional, enquanto para a Intoxicação Medicamentosa (IM), a dose usada é maior do que a dose normal e podem ocorrer acidentes. Relacionadas a vários eventos, como a própria administração, erros de dosagem, subtratamento, confusão entre medicamentos prescritos e ingestão involuntária ou intencional relacionada à administração errada ou acidental, abuso e tentativa ou autoagressão (SANTOS \& BOING,2018; PAMPLONA, et al., 2017).

Portanto, a intoxicação por medicamentos é causada por diversos mecanismos complexos, que podem estar relacionados às características individuais, farmacodinâmicas e processos farmacocinéticos de cada medicamento, podendo esses mecanismos ser afetados pelas propriedades farmacêuticas e desempenho do produto, interações com drogas e interações medicamentosas e alimentares (PAMPLONA, et al., 2017). 


\section{PAPEL DA INDÚSTRIA FARMACÊUTICA NAS INTOXICAÇÕES}

O grande número de medicamentos disponíveis e a comercialização ativa da indústria farmacêutica, aliados à falta de medidas preventivas e à fraca supervisão e controle das autoridades competentes, têm tornado as interações medicamentosas um problema crescente de saúde pública global. Outros fatores que podem agravar essa situação incluem: facilidade de acesso a medicamentos, erros de prescrição médica, maior dispensação de medicamentos e práticas de automedicação, levando ao Uso Racional de Medicamentos, múltiplos medicamentos, uso incorreto e abuso de psicotrópicos e antibióticos, além disso à falta de desenvolvimento efetivo da assistência farmacêutica, além dessas medidas, a segurança dos pacientes e a qualidade da assistência também estão em risco (GRETZLER et al.,2018).

As interações medicamentosas ocorrem quando os medicamentos são administrados em doses maiores do que as recomendadas para fins terapêuticos, preventivos e diagnósticos. Especialmente em crianças, essas intoxicações têm diferentes motivos, entre os quais podemos destacar: curiosidade nascida na idade, imaturidade fisiológica ( $\mathrm{CYP}_{450}$ ), aumento da sensibilidade aos efeitos tóxicos dos medicamentos, embalagens não lacradas, $O$ desconhecimento da mãe ou o menor enfoque na prevenção de acidentes aliado ao armazenamento incorreto, facilita a obtenção de medicamentos pelas crianças, é difícil calcular e medir com precisão a dosagem dos medicamentos, a prática de pais ou responsáveis auxiliarem na automedicação, a falta de anúncios sobre o perigo de overdose, o aparecimento de pílulas e a doçura da calda vão atrair crianças e ajudar no envenenamento acidental. (MAIOR et al., 2017).

\section{AUTOMEDICAÇÃO E O RISCO DE INTOXICAÇÃO}

No Brasil, esses medicamentos são considerados a principal causa de intoxicação, por mais seguro e inofensivo que pareça ser, podem causar inúmeras reações adversas. Em países desenvolvidos como França, Alemanha, Inglaterra, Itália, Estados Unidos, e Canadá, os medicamentos representam aproximadamente 50\% dos episódios de intoxicação registrados (CAIRES et al., 2018). 
A automedicação é prática definida como a administração ou uso de medicamentos sem prescrição ou orientação de profissional de saúde, com o objetivo de reduzir ou tratar sintomas ou mesmo promover saúde. Muitos desses medicamentos podem causar intoxicações além do uso de medicamentos com eficácia não comprovada, quando usados de forma inadequada, não observando a dosagem correta, o intervalo de dosagem e o tempo de uso. (FILHO \& JUNIOR, 2013).

A automedicação pode ser entendida como uma prática de risco à saúde e grave ameaça à saúde pública. Nesse caso, o farmacêutico deve orientar as pessoas a usarem os medicamentos de forma racional, visando reduzir os riscos e ser o mais eficaz possível (CHIAROT et al., 2010).

Segundo estatísticas da Associação Brasileira da Indústria Farmacêutica (Abifarma), cerca de 80 milhões de pessoas no Brasil são boas na automedicação, e esse índice está diretamente relacionado aos casos de intoxicação por medicamentos. Fatores como alto índice de autoadministração, baixo custo, fácil disponibilidade e falta de compreensão dos efeitos tóxicos dos medicamentos são as principais causas de intoxicações no Brasil, com graves consequências para a saúde pública (RANGEL \& FRANCELINO, 2018).

Outro agravante dessa prática, que tem relação positiva e agrava ainda mais essa realidade, é o hábito de deixar a medicação em casa, que é ocasionado pela incompletude ou interrupção do tratamento por motivos diversos. Essa prática se deve ao número excessivo de medicamentos prescritos, podendo também produzir reações adversas, aumentando ativamente os riscos de Uso Irracional de Medicamentos e Interação Medicamentosa, levando à deterioração da saúde (DOS SANTOS et al., 2018).

É muito comum a prática de armazenamento de medicamentos não utilizados em tratamentos anteriores, quando investigamos esse agravo à saúde da população, percebemos que essa prática está diretamente relacionada à influência familiar ou às prescrições antigas. Essa prática aparentemente inofensiva tem levado a uma proporção crescente de automedicação, risco de infarto do miocárdio e tentativas de suicídio (GALATO et al., 2012). 
Quando mencionamos o armazenamento inadequado de medicamentos, o paciente idoso merece mais atenção. Além da farmacocinética e da farmacodinâmica dos medicamentos, o Problemas Relacionados a Medicamentos, os erros de medicação e os problemas de identificação causados pelo consumo excessivo de medicamentos, tornam uma população mais susceptível a sofrerem com quadros de intoxicação (SILVA, 2016).

A prática da automedicação é um grave vício da sociedade brasileira, o baixo custo, fácil acesso e o desconhecimento dos riscos para saúde que os medicamentos podem produzir, por parte das pessoas que utilizam terapias medicamentosas são os principais responsáveis pelos altos índices de Interações Medicamentosas no Brasil, trazendo consequências violentas para saúde pública (RANGEL \& FRANCELINO, 2018).

Dados da OMS indicam que, anualmente mais de 4 milhões de pessoas no mundo são intoxicadas por medicamentos, o que retrata uma média anual de aproximadamente 1,5 a 3\% de intoxicados na população global. No Brasil, esses índices também são elevados, uma vez que, o país é o $5^{\mathrm{o}}$ maior consumidor mundial de medicamentos, ocupando a $\mathrm{I}^{\mathrm{a}}$ posição quando o assunto é restringido à América Latina.

\section{O RISCO DA AUTOMEDICAÇÃO}

O número crescente de casos registrados de Intoxicação Medicamentosa tem causado grande preocupação à saúde pública, devido à funcionalidade do sistema de saúde e à disponibilidade de medicamentos adquiridos, que estão diretamente relacionados à automedicação. Portanto, a assistência farmacêutica é fundamental para reduzir o número de casos de intoxicações por essa prática, sendo o farmacêutico um dos principais mediadores envolvidos na prevenção e promoção da saúde, pois mantém contato direto e frequente com a população (CHAVES, et al., 2017).

O efeito terapêutico do Uso Racional de Medicamentos é inegável, porém seu uso abusivo e irracional pode trazer riscos e algumas consequências para a saúde, como a Intoxicação Medicamentosa. Segundo dados da Organização Mundial da Saúde, nos países desenvolvidos os medicamentos respondem por até $15 \%$ dos gastos com saúde e 
nos países em desenvolvimento, em média, 35\% (DO ROCIO MALAMAN et al., 2013).

A automedicação é uma prática que pode ser definida como o uso de medicamentos sem receita e orientação médica. Nessa prática, o paciente decide qual medicamento usar com base nos sintomas, quase sempre por uma pessoa não qualificada, por exemplo, amigos, familiares ou funcionários da farmácia (CAIRES et al., 2018).

A prática da automedicação é o segundo maior motivo notificado pelo Intoxicação Medicamentosa, estando diretamente relacionado a interações medicamentosas, erros de dosagem, aparecimento de reações adversas e outras condições que constituem fatores de risco para intoxicações (KLINGER et al., 2016).

Vários fatores podem explicar a automedicação: a influência da indústria farmacêutica; investimento em publicidade; competição entre farmácias e laboratórios; prescrições empíricas; ineficiência no sistema público de saúde; e outros fatores que promovem o acesso das pessoas a todo e qualquer tipo de medicamento (CAIRES et al., 2018).

Portanto, é importante ressaltar que o farmacêutico desempenha um papel importante no processo de orientar o uso correto dos medicamentos, pois sua formação envolve um conhecimento aprofundado da área específica do assunto para garantir uma dispensação segura e um tratamento eficaz (SANTOS, 2017).

\section{AÇÕES DO FARMACÊUTICO NA EDUCAÇÃO EM SAÚDE}

Devido à grande importância que os medicamentos têm na garantia da saúde e bem-estar social, a discussão sobre políticas públicas específicas a realidade dos problemas relacionados às intoxicações, portanto, pautada e discutida em todas as esferas da saúde, que envolve uma equipe de protagonistas: legisladores, pacientes, empresários do ramo, publicitários e, principalmente, os profissionais de saúde (VIEIRA \& CAVEIÃO, 2016).

Como resultado de políticas públicas desapropriadas para preventivas ao Uso Irracional de Medicamentos, uma vez que, na maioria dos casos a intoxicação poderia ter sido evitada. Levando informações precisas e de qualidade à comunidade sobre a 
racionalização no uso de medicamentos que é uma importante ferramenta para alcançar mais níveis elevados de saúde (SILVA, 2018).

Os profissionais de saúde têm papel importante na prevenção do uso desordenado de medicamentos de forma a reduzir ou minimizar as suas consequências. Nesse contexto, o Farmacêutico, como profissional de saúde, assume papel principal no sistema de racionalização do consumo de medicamentos, de modo a manter maior eficácia, eficiência e segurança aos tratamentos medicamentosos, garantindo, dessa forma, melhor qualidade de vida aos pacientes (OLIVEIRA \& SILVA, 2014).

Outro conceito embutido na prática farmacêutica que enriquece o trabalho de prevenção de Interação Medicamentosa é a prática da Farmácia Clínica. Esse conceito surgiu durante os anos 6o nos Estados Unidos como alternativa para aprimorar os serviços prestados pelos profissionais da área como o objetivo de assegurar uma segurança e potencialização da farmacoterapia prescrita. Essa prática permitiu que o Farmacêutico, até então gestor, pudesse explorar seus conhecimentos clínicos para impedir eventuais adversidades pelo Uso Irracional de Medicamentos (SANTOS, 2017).

$\mathrm{Na}$ atualidade a Farmácia Clínica é um conceito mais amplo, que determina uma área da farmácia ligada na prática do Uso Racional de Medicamentos, de forma que o Farmacêutico acompanhe o paciente prestando-lhe a devida assistência assegurando uma farmacoterapia eficiente e livre de riscos à saúde.

Outros preceitos do profissional farmacêutico é o Cuidado Farmacêutico, na qual o Conselho Federal de Farmácia (CFF) descreve esse termo como um modelo de prática farmacêutica voltada ao ato de diversos serviços farmacêuticos conduzindo o paciente e seus familiares, bem como a comunidade alcançando a prevenção de adversidades procedentes da farmacoterapia (CONSELHO FEDERAL DE FARMÁCIA, 2016).

\section{CONCLUSÃO}

No Brasil a automedicação é uma atividade atual e frequente, principalmente em casos com venda livre de medicamentos. Analgésicos (substâncias que diminuem ou extinguem a dor) são os principais medicamentos consumidos pela população devido 
á motivos, como: econômicos e sociais, propiciados pela negligência e a má qualidade do atendimento e espera no Sistema Único de Saúde (SUS), que por muitas vezes aos impasses financeiros para à ida numa instituição privada a sociedade busca a flexibilização no atendimento em farmácias.

Entre esta atividade, pode ocorrer complicações graves, mesmo sendo mascarados alguns sintomas que provocam uma série de problemas de saúde.

Portanto, é função do farmacêutico pôr em prática seu conhecimento técnico e profissional, e com cautela orientar os clientes nas compras de medicamentos sem receita.

Diante desta pesquisa, é necessário destacar que intervenções no consumo responsável e consistente de medicamentos são essenciais, pois esta, é a verdade da antropologia, da automedicação para promover e proteger a saúde da população e da iniciativa privada.

Entretanto, a prescrição de medicamentos é comprovadamente um mecanismo de segurança a ponto de serem usados corretamente. Sendo assim, fica comprovado o relevante papel do farmacêutico como medida de prevenção ao uso indevido de medicamentos, por se tratar de um profissional que visa prestar serviços farmacêuticos de acordo com a legislação e a ética, contribuindo para que a sociedade tenha acesso aos medicamentos, orientação, ajuda qualificada, consciencialização dos perigos de comportamentos adversos, interações medicamentosas e intoxicações por remédios

\section{REFERÊNCIAS BIBLIOGRÁFICAS}

I- CAIRES, C. S.; BARONI, C. C.; PEREIRA, L. L. V. Intoxicação medicamentosa com foco nos efeitos do paracetamol. Revista Científica, v. I, n. I, 2018. Disponível em: http://revistas.unilago.edu.br/index.php/revista-cientifica/article/download/ro7/9o

2- CARVAlHO, A. F. Perfil epidemiológico dos casos de intoxicação por medicamentos registrados Centro de Informação e Assistência Toxicológica do Distrito Federal entre 20II e 2016. 75f.Trabalho de Conclusão de curso. UNB Faculdade de Ciências da Saúde, 2017. Disponível em: http://www.bdm.unb.br/handle/10483/18678 
3- Chaves, L. H. S.; VIANA, Á. C.; JÚNIOR, W. P. M.; LIMA, A.; DE CARVALHO, L. Intoxicação exógena por medicamentos: aspectos epidemiológicos dos casos notificados entre 2011 e 2015 no Maranhão. Revista Ciência \& SaberesFacema, v. 3, n. 2, p. 477-482, 2017. Disponível em: http://www.facema.edu.br/ojs/index.php/ReOnFacema/article/view/20

4- CHIAROT, R.; REBELLO, N. M.; RESTINI, C. B. A. A automedicação na cidade de Ribeirão Preto-SP e o papel do farmacêutico nessa prática. Revista Eletrônica Centro Científico Conhecer-Enciclopédia Biosfera [periódico na Internet], v. ıo, n. 6, 2010.

Disponível em:http://www.conhecer.org.br/enciclop/2010b/a\%20automedicacao.pdf

5- CONSELHO FEDERAL DE FARMÁCIA. Serviços farmacêuticos diretamente destinados ao paciente, à família e à comunidade: contextualização e arcabouço conceitual. Brasília: Conselho Federal de Farmácia, v. 200, 2016. Disponível em: http://www.cff.org.br/userfiles/Profar_Arcabouco_TELA_FINAL.pdf

6- CORRER, C. J.; OTUKI, M. F.; SOLER, O. Assistência farmacêutica integrada ao processo de cuidado em saúde: gestão clínica do medicamento. Revista Pan Amazônica de Saúde, v. 2, n. 3, p. 4I-49, 20II. Disponível em: http://scielo.iec.gov.br/scielo.php?pid=S2176$62232011000300006 \&$ script $=$ sci_arttext $\&$ thlng=es

7- DO ROCIO MALAMAN, K.; PARANAÍBA, A. S. C.; DUARTE, C. M. S.; CARDOSO, R. A. Perfil das intoxicações medicamentosas, no Brasil. InfarmaCiências Farmacêuticas, v. 2I, n. 7/8, p. 9-I5, 2013. Disponível em: http://revistas.cff.org.br/?journal=infarma\&page $=$ article\&op=view \&path\% ${ }_{5} \mathrm{~B} \% 5 \mathrm{D}=\mathrm{I}$ 34 
8- DOS SANTOS SANTANA, K. HORÁCIO, B. O., SILVA, J. E., JÚNIOR, C. D. A. C., GERON, V. L. M. G., TERRA JÚNIOR, A. T. O papel do profissional farmacêutico na promoção da saúde e do uso racional de medicamentos. Revista Científica da Faculdade de Educação e Meio Ambiente, v. 9, n. I, p. 399-412, 2018. Disponível em: http://repositorio.faema.edu.br:80oo/handle/123456789/1249

9- FILHO, P. C. P.; JÚNIOR, A. C. P. Automedicação em crianças de zero a cinco anos: fármacos administrados, conhecimentos, motivos e justificativas. Escola Anna Nery Revista de Enfermagem, v. 17, n. 2, p. 291-297, 2013. Disponível em: https://www.redalyc.org/pdf/1277/127728367013.pdf

Io- FUNDAÇÃO OSWALDO CRUZ. Sistema Nacional de Informações Tóxico Farmacológicas - SINITOX. Casos registrados de intoxicação e/ou envenenamento.20II [citado em io de março 2017]. Disponível em: 〈http://www.fiocruz.br/sinitox_novo/cgi/cgilua.exe/sys/start.htm?sid=349

II- GALATO, D.; MADALENA, J.; PEREIRA, G. B. Automedicação em estudantes universitários: a influência da área de formação. Ciência \& Saúde Coletiva, Santa Catarina, RS, [s.I], v.17, n.I2, p.3323-3330, 2012. Disponível em: https://www.scielosp.org/scielo.php?pid=Si413-

81232012001200017\&script=sci_arttext\&tlng=en

I2- GRETZler, V. S.; RODRIGUES, A. S.; VARGAS, D. A., PEREIRA, H. C., TERRA JÚNIOR, A. T. Atuação do farmacêutico no uso racional de medicamentos e na prevenção de intoxicação medicamentosa. Revista Científica da Faculdade de Educação e Meio Ambiente, v. 9, n. edesp, p. 547-550, 2018. Disponível em: http://www.faema.edu.br/revistas/index.php/Revista-FAEMA/article/view/58o

I3- KLINGER, E. I.; SCHMIDT, D. C.; LEMOS, D. B.; PASA, L.; POSSUELO, L. G.; DE MOURA VALIM, A. R. Intoxicação exógena por medicamentos na população jovem do Rio Grande do Sul. Revista de Epidemiologia e Controle de Infecção, v. I, n. 
I, p. 44-52, 2016. Disponível em: https://online.unisc.br/seer/index.php/epidemiologia/article/view/8216

II- LEITE SN, VIEIRA M, VEBER AP. Estudos de utilização de medicamentos: uma síntese de artigos publicados no Brasil e América Latina. Cien Saude Colet. $2008.13(\mathrm{I}): 793-802$.

I2- MAIOR, M. C. L. S.; OSORIO-DE-CASTRO, C. G. S.; ANDRADE, C. L. T. Internações por intoxicações medicamentosas em crianças menores de cinco anos no Brasil, 2003-2012. Epidemiologia e Serviços de Saúde, v. 26, p. 771-782, 2017. Disponível em: https://www.scielosp.org/article/ress/2017.v26n4/771-782/

13- MATHIAS, T. L.; GUIDONI, C. M.; GIROTTO, E. Tendências de eventos toxicológicos relacionados a medicamentos atendidos por um Centro de Informações Toxicológicas. Revista Brasileira de Epidemiologia, v. 22, p. ergoor8, 2019. Disponível em: https://www.scielosp.org/article/rbepid/2019.v22/ergoor8/pt/

I4- MONTE, B. S.; NUNES, M. S. T.; NUNES, M. D. D. S.; DE MELO MENDES, C. M. Estudo Epidemiológico das intoxicações por medicamentos registradas pelo Centro de Informação Toxicológica do Piauí-CITOX. Revista Interdisciplinar, v. 9, n. $3, \quad$ p. 2016. 96-104, Disponível em: https://dialnet.unirioja.es/servlet/articulo?codigo $=6772012$

15- NÓBREGA, H. O. S. Intoxicações por Medicamentos: Uma Revisão Sistemática com Abordagem nas Síndromes Tóxicas. Revista Saúde \& Ciência Online, v. 4, n. 2, p. I09-II9, 2015. Disponível em:http://www.ufcg.edu.br/revistasaudeeciencia/index.php/RSC-

UFCG/article/view/264

i6- OLIVEIRA, C. M.; SILVA, J. A. Papel do profissional farmacêutico no uso irracional de medicamentos em pacientes pediátricos. 2014. 27f. Trabalho de Conclusão 
de Curso - Fundação Universitária Vida Cristã, Pindamonhangaba, 2014. Disponível em:http://www.bibliotecadigital.funvicpinda.org.br:8o8o/jspui/handle/r23456789/27 2

17- PAMPlONA, M. H. A.; SARMENTO, W. M.; DOS SANTOS, D. F. Intoxicações medicamentosas em idosos. Editora realize. Anais V CIEH, pag. I - ıo, 2017. Disponível em: http://www.editorarealize.com.br/revistas/cieh/trabalhos/TRABALHO_EVo75_M D2_SAi5_ID727_16102017174950.pdf

I8- RANGEL, N. L.; FRANCELINO, E. V. Caracterização do Perfil das Intoxicações Medicamentosas no Brasil, durante 2013 a 2016. Id on line REVISTA DE PSICOLOGIA, v. I2, n. 42, p. I2I-I35, 2018. Disponível em: https://idonline.emnuvens.com.br/id/article/view/1302

I9- ROCHA, Andressa Ferreira. Automedicação no Brasil uma reflexão necessária. Faculdade de Educação e Meio Ambiente - FAEMA. Ariquemes-RO 2olr. Disponível em:

http://repositorio.faema.edu.br/bitstream/123456789/735/I/ROCHA\%2C\%20A.\%20 F.\%20\%20AUTORREMEDIA\%C3\%87\% 2oREFLEX\%C3\%83 O\%20NECESS\%C3\%8IRIA.pdf.

20- SANTOS, G. A. S.; BOING, A. C. Mortalidade e internações hospitalares por intoxicações e reações adversas a medicamentos no Brasil: análise de 2000 a 2014. Cadernos de Saúde Pública, v. 34, p. eooroogr7, 2018. Disponível em: https://www.scielosp.org/article/csp/2018.v34n6/eooroogr7/

21- SANTOS, J. S. Intervenções farmacêuticas na adesão ao tratamento farmacológico em usuários do centro de atenção psicossocial para álcool e outras drogas. Trabalho de Conclusão de Curso (Graduação em Farmácia) - Universidade Federal de Sergipe, 57fl. 2017. Disponível em: https://teses.ufs.br/handle/riufs/7454 
22- SILVA, L. T. D. C. Análise da automedicação, suas práticas e riscos sobre a saúde: revisão de literatura. 2016. 56f. Trabalho de Conclusão de Curso (Graduação em Farmácia) - Faculdade Maria Milza, 2or6. Disponível em: http://131.0.244.66:8082/jspui/handle/123456789/150

23- SILVA, T. J.; OLIVEIRA, V. B. Intoxicação medicamentosa infantil no Paraná. Visão Acadêmica, v. I9, n. I, 2018. Disponível em: https://revistas.ufpr.br/academica/article/view/57576

24- VIEIRA, D. M.; CAVEIÃO, C. Perfil das intoxicações medicamentosas no estado de São Paulo no período de 1999 a 2012 na perspectiva da vigilância sanitária. Revista Saúde e Desenvolvimento, v. 9, n. 5, p. II9-I4I, 2016. Disponível em: http://www.uninter.com/revistasaude/index.php/saudeDesenvolvimento/article/v iew/521 